\title{
Efecto de efluentes municipales en el crecimiento y contenido de lípidos de la microalga Scenedesmus acutus en laboratorio
}

\section{Effect of municipal effluents on the growth and lipid content of the microalgae Scenedesmus acutus in laboratory}

\author{
iD 'Elizabeth Vásquez de la Cruz (iD) 'Tatiana Zavaleta Gámez (iD ' Sorayda Mendoza Espinoza \\ iD 'Juan Fernando Merino Moya
}

Universidad Nacional del Santa. Chimbote - Perú. ${ }^{1}$

\section{Resumen}

El objetivo de este estudio fue evaluar el efecto de las aguas residuales municipales (ARM) de la laguna de estabilización secundaria "Las Gaviotas", (Nuevo Chimbote, Ancash-Perú). sobre el crecimiento poblacional y contenido de lípidos de la microalga Scenedesmus acutus. Los ensayos fueron realizados por triplicado dosificando cultivos de S. acutus con 25, 50, 75 y $100 \%$ de ARM y un cultivo control con HM (Merino, 1999) durante siete días. Las concentraciones 50, 75 y $100 \%$ de ARM alcanzaron mayores tasas de crecimiento (u) que el control $(0,461$; 0,$467 ; 0,479$ y 0,453 día-1, respectivamente) y elevados contenidos de lípidos $(24,47 ; 23,94 ; 23,64$ y $21,78 \%)$, para los cultivos dosificados con 50, 75, $100 \%$ de ARM y control, respectivamente en el quinto día. El contenido de nitrógeno fue menor en los cultivos dosificados con ARM. Los cultivos de S. acutus dosificados con ARM presentaron altas tasas de crecimiento y contenido de lípidos que los controles, debido al menor contenido de nitrógeno.

Palabras clave: Microalga, Scenedesmus acutus, crecimiento poblacional, lípidos, nitrógeno.

Recibido: Junio 2020

Aceptado: Octubre 2020

\section{Abstract}

The aim of this study was to evaluate the effect of municipal wastewater (MRA) from the secondary stabilization lagoon "Las Gaviotas", (Nuevo Chimbote, Ancash-Peru). on population growth and lipid content of the microalgae Scenedesmus acutus. The tests were carried out in triplicate, dosing S. acutus cultures with 25, 50, 75 and $100 \%$ MRA and a control culture with HM (Merino, 1999) for seven days. The 50,75 and $100 \%$ concentrations of MRA reached higher growth rates $(u)$ than the control $(0.461,0.467,0.479$ and 0.453 day-1, respectively) and high lipid contents $(24.47,23.94,23,64$ and $21.78 \%)$, for the cultures dosed with 50, 75, $100 \%$ MRA and control, respectively on the fifth day. Nitrogen content was lower in cultures dosed with MRA. The cultures of S. acutus dosed with MRA presented higher growth rates and lipid content than the controls due to the lower nitrogen content. 
Keywords: Microalgae, Scenedesmus acutus, municipal effluent, population growth, lipids, nitrogen.

\section{INTRODUCCIÓN}

Los inadecuados manejos de efluentes o aguas residuales causan serios problemas al ambiente, la salud humana y el desarrollo económico (PNUMA., 2004), cuya problemática se ha incrementado en relación a su tratamiento y posible reutilización (Méndez et al., 2010), mediante la aplicación de tecnologías sostenibles desde la captación de aguas, su tratamiento y su reutilización (Morató et al., 2006). Los efluentes industriales o municipales deben ser tratadas antes de descargarlos en el sistema del alcantarillado municipal a fin de evitar malos olores, reducir el contenido de sólidos suspendidos, de compuestos orgánicos biodegradables y los microorganismos patógenos (Valdez \& Vázquez., 2003).

Actualmente, los efluentes o aguas residuales municipales de Nuevo Chimbote son recepcionados en las "lagunas de oxidación" Las Gaviotas, Villa María y Laguna Centro Sur A "tratando" solamente el 37 \% (SUNASS., 2005), generando afloramientos que al salinizar los terrenos aledaños las inhabilita para cualquier actividad agrícola (Loayza, 2002). Además, el deficiente funcionamiento de la infraestructura del tratamiento ocasiona la salida de los efluentes hacia el mar contaminando diversas especies marinas utilizadas en la alimentación humana (BCEOM \& OIST., 1998), por lo que el tratamiento de los efluentes municipales debe priorizarse mediante métodos biológicos, físicos y químicos (Blackall et al., 2002; Malick., 2002).

El tratamiento de efluentes o aguas residuales con microalgas es una buena alternativa para remover compuestos orgánicos e inorgánicos y reducir la materia orgánica (Zamani et al., 2011), y generar biomasa algal y efluentes de buena calidad con altas concentraciones de oxígeno (González, 2006), con efectos positivos en la salubridad del ambiente acuático.

La microalga clorofita Scenedesmus es propia de cuerpos de agua dulce y se presenta como célula solitaria o formando colonias denominadas cenobios (Garibay et al., 2009), que por su gran capacidad de adaptación ecofisiológica y plasticidad bioquímica son consideradas maquinarias fotosintéticas (Escudero., 2012) y por su elevada actividad metabólica, son capaces de soportar las elevadas concentraciones de nutrientes presentes en aguas residuales, generando biomasa, el cual es importante en la depuración del ambiente (Herbas et al., 2006).

El uso de efluentes municipales, por contener nitrógeno, fósforo, potasio y otros nutrientes, permite el cultivo de Scenedesmus acutus para generar biomasa algal a bajo costo y reciclar alrededor del $90 \%$ de dichos nutrientes, sin competir con la producción de alimentos producidos por la agricultura (Yang et al., 2011). Adicionalmente, la biomasa puede ser utilizada en la obtención de proteínas, lípidos, pigmentos, etc. de importante valor económico.

El principal objetivo fue evaluar el efecto de diferentes concentraciones del efluente municipal sobre el crecimiento poblacional y contenido de lípidos de la microalga Scenedesmus acutus en laboratorio.

\section{MATERIALES Y MÉTODOS}

El presente trabajo se realizó en el Laboratorio de Cultivo de Especies Auxiliares de la Universidad Nacional del Santa. El cultivo inóculo de Scenedesmus acutus fue proporcionado por este laboratorio en volúmenes suficientes y buenas condiciones de salubridad. Todos los cultivos ensayados fueron agitados e iluminados constantemente con dos fluorescentes de luz blanca de $40 \mathrm{w}$.

Los cultivos controles fueron dosificados con medio de cultivo HM (Merino, 1999) elaborado con fertilizantes agrícolas de uso convencional, para el cultivo masivo al aire libre de 
S. acutus (Tabla 1), mientras que el medio de cultivo experimental fue preparado mediante la cocción a $100^{\circ} \mathrm{C}$ por 30 min de las aguas residuales municipales (ARM), procedente de la "laguna de oxidación" "Las Gaviotas", ubicada en Nuevo Chimbote (Ancash-Perú), que lue- go de enfriado y filtrado con de 5 micras, fue autoclavado por 15 min a $121^{\circ} \mathrm{C}$ para eliminar microorganismos patógenos (Tabla 2). Los ensayos fueron realizados por triplicado dosificando cultivos de S. acutus con 25, 50, 75 y $100 \%$ de ARM y un cultivo control con HM.

\section{Tabla 1}

Composición química del medio HM.

\begin{tabular}{cc}
\hline COMPONENTE & CONCENTRACIÓN $\left(\mathrm{mg} \mathrm{L}^{-1}\right)$ \\
\hline Urea & 206,0 \\
$\mathrm{H}_{3} \mathrm{PO}_{4}$ & 19,0 \\
$\mathrm{KCl}$ & 30,0 \\
$\mathrm{FeCl}_{3}$ & 2,5 \\
\hline
\end{tabular}

\section{Tabla 2}

Componentes de los tratamientos utilizados en el cultivo de Sc. acutus con diferentes concentraciones de aguas residuales municipales (ARM).

\begin{tabular}{cccccc}
\hline \multirow{2}{*}{ PARÁMETROS } & \multicolumn{5}{c}{ TRATAMIENTOS } \\
\cline { 2 - 6 } & T0 $(0 \%)$ & $\begin{array}{c}\text { T1 } \\
(25 \%)\end{array}$ & $\begin{array}{c}\text { T2 } \\
(50 \%)\end{array}$ & $\begin{array}{c}\text { T3 } \\
(75 \%)\end{array}$ & $\begin{array}{c}\text { T4 } \\
(100 \%)\end{array}$ \\
\hline Agua Residual $(\mathrm{mL})$ & 0 & 250 & 500 & 750 & 900 \\
Agua Potable $(\mathrm{mL})$ & 900 & 750 & 400 & 150 & 0 \\
Inóculo $(\mathrm{mL})$ & 100 & 100 & 100 & 100 & 100 \\
\hline TOTAL $(\mathrm{mL})$ & 1000 & 1000 & 1000 & 1000 & 1000 \\
\hline
\end{tabular}

Los cultivos de Sc. acutus se realizaron en botellas plásticas de $2000 \mathrm{~mL}$ de volumen total con $1000 \mathrm{~mL}$ de volumen efectivo conteniendo las diferentes proporciones del efluente municipal (EM) cuyos principales parámetros se presentan en la Tabla 3 y los contenidos de nitrógeno y fósforo en cada unidad experimental en la Tabla 4.

\section{Tabla 3}

Parámetros del efluente municipal (EM).

\begin{tabular}{|c|c|}
\hline PARÁMETROS & CONCENTRACIÓN \\
\hline Humedad (\%) & 86,39 \\
\hline Salinidad (ups) & 2,73 \\
\hline pH (unid.) & 7,14 \\
\hline Sólidos Totales $\left(\mathrm{mg} \mathrm{L}^{-1}\right)$ & 136,12 \\
\hline Nitratos $\left(\mathrm{mg} \mathrm{L}^{-1}\right)$ & 19,15 \\
\hline Nitritos $\left(\mathrm{mg} \mathrm{L}^{-1}\right)$ & 0,28 \\
\hline Amonio (mg L $\left.\mathrm{L}^{-1}\right)$ & 5,14 \\
\hline Fosfatos $\left(\mathrm{mg} \mathrm{L}^{-1}\right)$ & 4,03 \\
\hline
\end{tabular}




\section{Tabla 4}

Contenido de agua residual, nitrógeno y fósforo del efluente municipal (EM) según los diferentes tratamientos.

\begin{tabular}{cccccc}
\hline \multirow{2}{*}{ PARÁMETROS } & \multicolumn{5}{c}{ TRATAMIENTOS } \\
\cline { 2 - 6 } & $\begin{array}{c}\text { T0 }(\mathrm{HM}) \\
(0 \%)\end{array}$ & $\begin{array}{c}\text { T1 } \\
(25 \%)\end{array}$ & $\begin{array}{c}\text { T2 } \\
(50 \%)\end{array}$ & $\begin{array}{c}\text { T3 } \\
(75 \%)\end{array}$ & $\begin{array}{c}\text { T4 } \\
(90 \%)\end{array}$ \\
\hline Agua Residual $(\mathrm{mL})$ & 0 & 250 & 500 & 750 & 900 \\
Nitrógeno $\left(\mathrm{mg} \mathrm{L}^{-1}\right)$ & 100 & 2,22 & 4,44 & 6,66 & 7,99 \\
Fósforo $\left(\mathrm{mg} \mathrm{L}^{-1}\right)$ & 6,14 & 1,01 & 2,02 & 3,02 & 3,63 \\
\hline
\end{tabular}

Se registraron diariamente durante los siete días del cultivo el $\mathrm{pH}$ y la temperatura, haciendo uso de un $\mathrm{pH}$-metro digital $( \pm 0,01)$ con termómetro $\left( \pm 0,1^{\circ} \mathrm{C}\right)$ marca Oakton y la determinación del crecimiento poblacional con cámara Neubauer y un microscopio binocular marca Olympus ${ }^{\oplus}$, cuyos valores resultantes permitieron la elaboración de las curvas de crecimiento poblacional y se determinaron la tasa de crecimiento poblacional diaria $(\mu)$ mediante la fórmula de Guillard (1975):

$$
\mu=\frac{\ln \left(N_{f} / N_{0}\right)}{T_{f}-T_{0}}
$$

Donde $N_{0}$ y $N_{f}$ corresponden al número de células por $\mathrm{mL}$ en los tiempos $T_{0}$ y $T_{f}$ respectivamente.

La determinación del contenido de lípidos en Sc. acutus se realizó al llegar a la fase exponencial de acuerdo a la técnica de Blight \& Dyer (1959) y el contenido porcentual según la metodología modificada de Marsh \& Weinsteinn (1966), aplicando la siguiente ecuación:

$$
L(\%)=\frac{\left(\left(\frac{\text { Absorbancia }}{P}\right) \times V\right)}{M} \times 100
$$

Donde:

V: Volumen final de muestra analizada (15 $\mathrm{mL})$.

M: Peso seco (mg) en $10 \mathrm{~mL}$ de cultivo.

P: Promedio de las pendientes de las curvas de calibración con aceite de oliva y colesterol $(4,4714)$.
El contenido de lípidos ( $\left.\mathrm{mg} \mathrm{L}^{-1}\right)$ de cada tratamiento fue determinado utilizando la siguiente fórmula:

$$
L B\left(m g L^{-1}\right)=\frac{L x B}{100}
$$

Donde:

L: Porcentaje de lípidos (\%).

B: Biomasa seca por litro (mg).

\section{Análisis estadístico de los datos}

Los datos del crecimiento poblacional y contenido de lípidos fueron sometidos al análisis de varianza (ANOVA) para establecer diferencias entre sus promedios y se utilizó la prueba de Tukey HSD, para establecer el mejor promedio, con un nivel de significancia de $\mathrm{p}:<$ 0,05, para ambos casos. El tratamiento estadístico fue desarrollado utilizando los programas, Office Excel 2013 y SPSS 20.0 para Microsoft Windows 8.

\section{RESULTADOS}

\section{pH y temperatura de los cultivos}

Los valores del pH de los cultivos de Sc. acutus variaron de 7,81 a 9,64 y la temperatura de 23,4 a $25,1^{\circ} \mathrm{C}$ durante los siete días del cultivo; siendo estadísticamente similares $(p>0,05)$ en cada uno de los días que duró el experimento.

\section{Crecimiento poblacional de S. acutus}

En la Tabla 5 y fig. 2 se observan las variaciones en las densidades poblacionales de los cultivos de S. acutus cultivados con medio ARM y control. 


\section{Tabla 5}

Densidad poblacional (x106 cél. mL-1) de Sc. acutus cultivados dosificados con medio ARM y control (HM).

\begin{tabular}{cccccc}
\hline DíA DE & \multicolumn{5}{c}{ ARM (\%) } \\
\cline { 2 - 6 } CULTIVO & CONTROL & $\mathbf{2 5}$ & $\mathbf{5 0}$ & $\mathbf{7 5}$ & $\mathbf{1 0 0}$ \\
\hline $\mathbf{0}$ & $2,05 \pm 0,00^{\mathrm{a}}$ & $2,05 \pm 0,00^{\mathrm{a}}$ & $2,05 \pm 0,00^{\mathrm{a}}$ & $2,05 \pm 0,00^{\mathrm{a}}$ & $2,05 \pm 0,00^{\mathrm{a}}$ \\
$\mathbf{1}$ & $2,93 \pm 0,28^{\mathrm{a}}$ & $3,04 \pm 0,16^{\mathrm{a}}$ & $3,10 \pm 0,14^{\mathrm{a}}$ & $2,95 \pm 0,25^{\mathrm{a}}$ & $3,32 \pm 0,44^{\mathrm{a}}$ \\
$\mathbf{2}$ & $4,84 \pm 0,85^{\mathrm{a}}$ & $5,56 \pm 0,54^{\mathrm{a}}$ & $5,82 \pm 0,59^{\mathrm{a}}$ & $5,45 \pm 0,51^{\mathrm{a}}$ & $5,45 \pm 0,53^{\mathrm{a}}$ \\
$\mathbf{3}$ & $9,17 \pm 0,76^{\mathrm{b}}$ & $9,86 \pm 0,89^{\mathrm{ab}}$ & $11,50 \pm 0,58^{\mathrm{a}}$ & $10,75 \pm 0,28^{\mathrm{ab}}$ & $10,46 \pm 0,68^{\mathrm{ab}}$ \\
$\mathbf{4}$ & $15,45 \pm 0,71^{\mathrm{a}}$ & $12,58 \pm 0,72^{\mathrm{b}}$ & $15,97 \pm 0,78^{\mathrm{a}}$ & $15,99 \pm 0,86^{\mathrm{a}}$ & $16,55 \pm 0,93^{\mathrm{a}}$ \\
$\mathbf{5}$ & $19,74 \pm 1,38^{\mathrm{b}}$ & $13,95 \pm 0,57^{\mathrm{c}}$ & $20,56 \pm 0,67^{\mathrm{ab}}$ & $21,19 \pm 1,13^{\mathrm{ab}}$ & $22,47 \pm 0,85^{\mathrm{a}}$ \\
$\mathbf{6}$ & $21,21 \pm 0,95^{\mathrm{b}}$ & $13,87 \pm 1,33^{\mathrm{c}}$ & $22,32 \pm 0,89^{\mathrm{ab}}$ & $23,65 \pm 0,64^{\mathrm{ab}}$ & $24,04 \pm 1,05^{\mathrm{a}}$ \\
$\mathbf{7}$ & $23,83 \pm 0,67^{\mathrm{a}}$ & $14,06 \pm 0,63^{\mathrm{b}}$ & $22,92 \pm 1,04^{\mathrm{a}}$ & $24,51 \pm 0,73^{\mathrm{a}}$ & $24,93 \pm 0,86^{\mathrm{a}}$
\end{tabular}

Letras diferentes en la misma fila, indica diferencia significativa $(\mathrm{p}:<0,05)$.

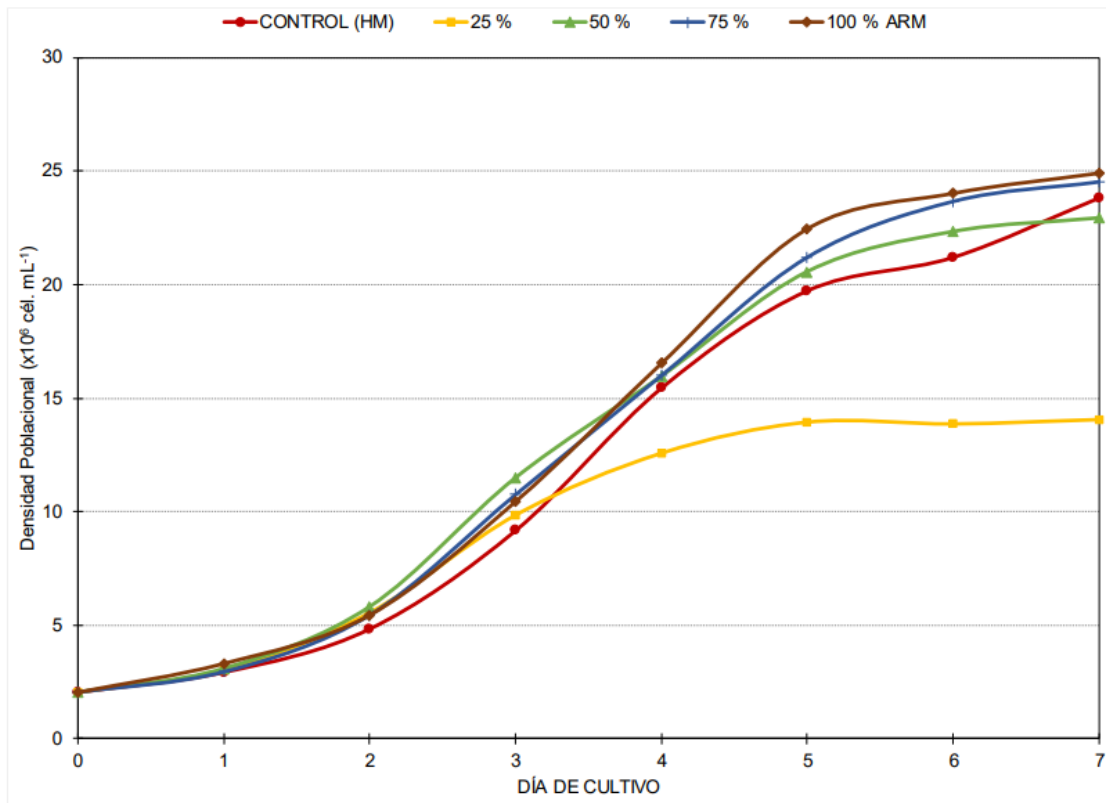

Figura 2. Curvas de crecimiento poblacional de S. acutus cultivados con medio ARM.

Los cultivos de S. acutus se iniciaron con densidades poblacionales significativamente similares $(p>0,05)$ de $2,05 \times 10^{6}$ cél. $\mathrm{mL}^{-1}$ e incrementaron diariamente con tendencias similares $(p>0,05)$ hasta el segundo día de cultivo. A partir del tercer día, los mayores promedios poblacionales fueron significativos $(p:<0,05)$ y determinados en los trata- mientos con 50,75 y $100 \%$ y los menores en los de $25 \%$ de ARM (Tabla 5).

Los mejores valores de la tasa de crecimiento $(\mu)$ de los cultivos de $S$. acutus dosificados con ARM fueron determinados en el 5to día y correspondieron a los dosificados con 50, 75 y $100 \%$ de ARM (Tabla 6). 
Tabla 6

Tasa de crecimiento $(\mu)$ de Sc. acutus en los cultivados con ARM y control (HM) en el 5 to día.

\begin{tabular}{cccccc}
\hline \multirow{2}{*}{ PARÁMETRO } & \multicolumn{5}{c}{ ARM (\%) } \\
\cline { 2 - 6 } & CONTROL & $\mathbf{2 5}$ & $\mathbf{5 0}$ & $\mathbf{7 5}$ & $\mathbf{1 0 0}$ \\
\hline $\mathbf{N}_{\mathbf{0}}$ & $2,05 \pm 0,00$ & $2,05 \pm 0,00$ & $2,05 \pm 0,00$ & $2,05 \pm 0,00$ & $2,05 \pm 0,00$ \\
$\mathbf{N}_{\mathbf{f}}$ & $19,74 \pm 1,38$ & $13,95 \pm 0,57$ & $20,56 \pm 0,67$ & $21,19 \pm 1,13$ & $22,47 \pm 0,85$ \\
$\left.\boldsymbol{\mu} \mathbf{( d}^{-\mathbf{1}}\right)$ & $0,453 \pm 0,013^{\mathrm{b}}$ & $0,383 \pm 0,008^{\mathrm{c}}$ & $0,461 \pm 0,007^{\mathrm{ab}}$ & $0,467 \pm 0,011^{\mathrm{ab}}$ & $0,479 \pm 0,007^{\mathrm{a}}$ \\
& & & \\
\hline
\end{tabular}

\section{Contenido de lípidos en Sc. acutus}

Todos los cultivos de S. acutus dosificados con ARM presentaron mayores valores de lípidos que los controles, siendo más elevado en los dosificados con $25 \%$ (27,98\%); sin embargo, el contenido promedio de lípidos en biomasa ( $\mathrm{mg} \mathrm{L}^{-1}$ ) fue estadísticamente similar con 50, 75 y $100 \%$ de ARM y entre los cultivos control y dosificados con 25\% de ARM (Tabla 7).

\section{Tabla 7}

Contenido de lípidos totales (\%) y biomasa de lípidos (mg L-1) de Sc. acutus en el 5 to día de cultivo.

\begin{tabular}{|c|c|c|c|c|c|}
\hline \multirow{2}{*}{ PARÁMETROS } & \multicolumn{5}{|c|}{ ARM (\%) } \\
\hline & $\overline{\text { CONTROL (HM) }}$ & 25 & 50 & 75 & 100 \\
\hline Biomasa ( $\left.\mathrm{mg} \mathrm{L}^{-1}\right)$ & $680,0 \pm 47,3$ & $510,0 \pm 20,9$ & $752,0 \pm 24,2$ & $775,3 \pm 41,1$ & $821,3 \pm 30,6$ \\
\hline Lípidos ( \% ) & $21,78 \pm 0,49^{\mathrm{c}}$ & $27,98 \pm 0,65$ & $24,47 \pm 0,44^{b}$ & $23,94 \pm 0,46^{\mathrm{b}}$ & $23,64 \pm 0,51^{\mathrm{b}}$ \\
\hline Lípidos ( mg L $^{-1}$ ) & $148,3 \pm 13,7^{\mathrm{b}}$ & $142,8 \pm 9,1^{b}$ & $184,1 \pm 9,2^{\mathrm{a}}$ & $185,7 \pm 13,3^{\mathrm{a}}$ & $194,3 \pm 11,3^{\mathrm{a}}$ \\
\hline
\end{tabular}

Letras diferentes en la misma fila, indica diferencia significativa $(\mathrm{p}:<0,05)$.

\section{DISCUSIÓN}

El crecimiento de los cultivos algales es influenciado por diversos factores tales como la temperatura, $\mathrm{pH}$, salinidad, fotoperiodo, agitación, concentración de nutrientes, etc., de tal manera que el éxito de tales cultivos consiste en proporcionar adecuadas condiciones de crecimiento y de nutrientes, especialmente nitrógeno y fósforo.
Respecto a la temperatura, las microalgas crecen bien en determinado rango que varía según la especie algal y suele estar por debajo de los $25^{\circ} \mathrm{C}$ o soportar hasta los $36^{\circ} \mathrm{C}$ (Abalde et al.; 1995), de tal manera que los valores determinado de $23,4{ }^{\circ} \mathrm{C}$ a $25,1^{\circ} \mathrm{C}$ están dentro de este rango; asimismo, las variaciones del $\mathrm{pH}(7,81$ y 9,64) concuerdan con Richmond \& 
Becker (1986), quienes consideran que varían entre 7 y 8 y que el incremento en algunas unidades no afecta su crecimiento. En consecuencia, las variaciones de temperatura y $\mathrm{pH}$ de los cultivos algales no han afectado los resultados obtenidos.

Los nutrientes, inorgánicos y orgánicos, presentes en el medio de cultivo ARM derivados de la degradación de la materia orgánica, permiten el crecimiento sostenido de S. acutus por su capacidad de crecer en medios utilizando residuos de la pesca y maricultura (Merino et al., 2003; Fernández \& Paredes, 2007; Ipanaqué \& Paredes, 2009). También Wong et al. (2015), demostraron la capacidad de Chlorella sp. para remover el nitrógeno ( $\mathrm{N}$ ) y fósforo (P) de aguas residuales diluidas al $50 \%$. Las concentraciones de fósforo y nitrógeno en el ARM permitieron altos crecimientos de S. acutus, en comparación a lo reportado por Chacón et al. (2004) en Scenedesmus sp. utilizando agua residual filtrada $\left(9,35 \times 10^{6}\right.$ cél. $\left.\mathrm{mL}^{-1}\right)$ y medio sintético $\left(4,69 \times 10^{6}\right.$ cél. $\left.\mathrm{mL}^{-1}\right)$ menores a los reportados en los tratamientos con $50 \%$, $75 \%$ y $100 \%$ de ARM.

En relación a la tasa de crecimiento, los valores reportados por Martínez et al. (2000) de $1,08 d^{-1}$ y $4,56 d^{-1}$ en S. obliquus utilizando aguas residuales procedente del tratamiento secundario y suministración continua de $\mathrm{CO}_{2}$; por Ma et al. (2012) de 0,44 $\mathrm{d}^{-1}$ en cultivos de la cepa de Scenedesmus sp. LX1 en aguas residuales, y 0,24 $\mathrm{d}^{-1}$ por Durvasula et al. (2015) en Scenedesmus sp. cultivadas con aguas residuales domésticas enriquecidas con $1 \%$ de Tris-Acetato-fosfato, son menores a los obtenidos con ARM en el presente ensayo.

El menor crecimiento obtenido con $25 \%$ de ARM, puede ser explicado por la deficiencia de nitrógeno y fósforo que hacen más lentos la fotosíntesis, estableciéndose una relación inversa entre la tasa de crecimiento y la cantidad de nitrógeno; sin embargo, a partir de 50 $\%$ de ARM el crecimiento mejora por el mayor aporte de $\mathrm{N}$ y P orgánicos derivados de la muerte y desintegración bacteriana como consecuencia de las condiciones altamente aeróbicas de los cultivos algales creciendo vigorosamente.

Se ha reportado que la calidad y cantidad de nutrientes influyen en la composición química de las microalgas propiciando cambios en su crecimiento y composición química (Henriksen et al., 2002; Beopoulos et al., 2009) y que el contenido de puede llegar hasta 90 $\%$ en peso seco dependiendo de la especie y las condiciones ambientales del cultivo (Spolaore et al., 2006; Beopoulos et al., 2009). La acumulación de lípidos está asociada con el crecimiento poblacional y la edad del cultivo, así tenemos que Nannochloropsis oculata, cultivada en un medio limitado en nitrógeno presentan porcentajes de lípidos de 30,8\%, $39,7 \%$ y $50,4 \%$ para las fases logarítmica, estacionaria temprana, y estacionaria final, respectivamente (Chiu et al., 2009).

Los resultados demostraron que los cultivos dosificados con $25 \%$ de ARM presentan mayor contenido de lípidos por presentar menor concentración de nitrógeno; sin embargo, las tasas de crecimiento en los cultivos controles que contienen mayor concentración de nitrógeno, obtienen menor concentración de lípidos y menor tasa de crecimiento que los cultivos dosificados con ARM, que podría explicarse por la mayor variedad y complejidad de sustancias químicas, orgánicas e inorgánicas, presentes en las aguas residuales. Adicionalmente, cabe la posibilidad de la existencia de algún estrés colateral derivado de la presencia de bacterias anaeróbicas, al inicio de los cultivos, y/o la muerte posterior de tales microorganismos.

En condiciones limitadas de nitrógeno, el metabolismo celular se orienta a la formación y acumulación de las sustancias de reserva (hidratos de carbono y lípidos), como respuesta a una condición severa de estrés, ya que el nitrógeno es constituyente principal de proteínas y ácidos nucleicos que son altamente 
abundante en las células vivas (Jiang, 2002; Huang et al., 2003).

Wong et al. (2015), trataron aguas residuales domésticas primarias y filtradas con Scenedesmus quadricauda, encontrando las mayores concentraciones de microalgas aclimatadas y no aclimatadas de 0,995 $\mathrm{g} \mathrm{L}^{-1}$ y $0,940 \mathrm{~g} \mathrm{~L}^{-1}$, respectivamente, y al quinto día fue removido el $90 \%$ de ortofosfato y $95 \%$ de nitrógeno amoniacal, en cultivos aclimatados y no aclimatados, respectivamente; con niveles de lípidos mayores al $13 \%$ para todos los tratamientos, siendo el más alto a una concentración del $50 \%$ de aguas residuales con 18,1\% de lípidos, y $80 \%$ de los fosfatos fue removido en concentraciones de aguas residuales de 25 y $50 \%$ a seis días de cultivo; asimismo el 90 \% del nitrógeno amoniacal fue removido, en ambos casos. Es posible utilizar aguas residuales municipales en el cultivo masivo de microalgas para la obtención de lípidos aprovechando la disponibilidad de nutrientes que permiten sostener el metabolismo de ciertos microorganismos fotosintéticos y la generación del oxígeno producido oxidará los compuestos residuales propiciando la depuración eficiente de las aguas residuales (Chacón et al., 2004; Shin et al., 2015).

\section{CONCLUSIONES}

Las variaciones de la temperatura y $\mathrm{pH}$ de los cultivos de S. acutus, por estar dentro de los rangos adecuados no interfirieron en los resultados obtenidos.

Las aguas residuales municipales tratadas térmicamente y utilizada como medio de cultivo (ARM) permiten obtener mejores crecimientos y contenidos de lípidos que los controles dosificado con HM (Merino, 1999), medio desarrollado para el cultivo masivo de S. acutus; asimismo, todos los cultivos de S. acutus dosificados con concentraciones de 50, 75 y 100 $\%$ de ARM no presentaron diferencias estadísticamente significativas entre ellas.

\section{REFERENCIAS BIBLIOGRÁFICAS}

Abalde, J.; A. Cid; P. Fidalgo; E. Torres \& C. Herrero. (1995). Microalgas: Cultivos y Aplicacio- nes. Monografía No 26. Coruña: Laboratorio de Microbiología. Facultad de Ciencias, Universidad de Coruña. La Coruña, España. 181 p

Andrade, C.; A. Vera; C. Cárdenas \& E. Morales. (2009). Biomass production of microalgae Scenedesmus sp. with wastewater from fishery. Rev. Tec. Ing. Univ. Zulia. 32(2):126-134.

BCEOM \& OIST (Asociados). (1998). Estudios definitivos de la primera etapa de ampliación de los sistemas de agua potable y alcantarillado de la localidad de Chimbote. Minist. Presid. Chimbote, Perú.

Beopoulos, A.; T. Chardot \& J. Nicaud. (2009) Yarrowia lipolytica: a model and a tool to understand the mechanisms implicated in lipid accumulation. Biochimie. 91(6):692-696.

Blackall, L.; G. Crocetti; A. Saunders \& P. Bond. (2002) A review and update of the microbiology of enhanced biological phosphorus removal in wastewater treatment plants. Antonie Leeuwenhoek. 81:681-692.

Bligh, E. \& W. Dyer. (1959) A rapid method of total lipid extraction and purification. Can. J. Biochem. Physiol. 3:911-917.

Chacón, C.; C. Andrade; C. Cárdenas; I. Araujo \& E. Morales (2002). Uso de Chlorella sp. y Scenedesmus sp. en la remoción de nitrógeno, fósforo y DQO de aguas residuales urbanas de Maracaibo, Venezuela. Boletín del Centro de Investigaciones Biológicas, Universidad del Zulla, Maracaibo, Venezuela. 38(2):94-108.

Chiu, S.; C. Kao; M. Tsai; S. Ong; C. Chen \& C. Lin. (2009). Lipid accumulation and $\mathrm{CO}_{2}$ utilization of Nannochloropsis oculata in response to $\mathrm{CO}_{2}$ aeration. Bioresource Technol. 100(2):833-838.

Durvasulaa, R.; I. Hurwitza; A. Fiecka \& D.V. Raoa. (2015) Culture, growth, pigments and lipid content of Scenedesmus species, an extremophile microalga from Soda Dam, New Mexico in wastewater. Algal Research. 10:128133. 
Escudero, R. (2012). Planta de producción de microalgas con fines energéticos. Proyecto fin de Carrera de Ingeniería Química. Universidad de Almería. Almería, España. 342p.

Fernández, M. \& C. Paredes. (2007). Efecto del extracto de ensilado de pescado y urea en el crecimiento poblacional y contenido de carbohidratos y lípidos de la microalga Tetraselmis suecica cultivada en condiciones de laboratorio. Tesis para optar el Título de Biólogo Acuicultor. Universidad Nacional del Santa. Nuevo Chimbote, Perú. 62p.

Garibay, A., R, Vázquez-Duhalt; M. Sánchez; L. Serrano \& A. Martínez. (2007). Biodiesel a partir de microalgas. BioTecnología. 13(3):38-61.

González, M. 2006. Aplicación e importancia de las microalgas en el tratamiento de aguas residuales. Contactos. 59:64-70.

Henriksen, P.; B. Riemann; H. Kaas; H. Munk \& H. Lang. (2002). Effects of nutrient limitation and irradiance on marine phytoplankton Pigments. J. Plank. Res. 24(9):835-858.

Herbas, R.; F. Rivero \& A. Gonzales. (2006). Indicadores biológicos de calidad del agua. Tesis de Maestría en Ingeniería Ambiental. Universidad Mayor de San Simón. Cochabamba, Bolivia. 21p.

Huang, X.; H. Zhou; C. Yuan \& M. Sun. (2006). The effects of nitrogen source and concentration on the nutritional value of Nannochloropsis oculata. J. Shanghai Fish Univ. 12:113-116.

Ipanaqué, J. \& I. Paredes. (2009). Efecto del ensilado de los desechos blandos de Argopecten purpuratus "concha de abanico", en el crecimiento poblacional y contenido de lípidos totales de Tetraselmis suecica, en condiciones de laboratorio. Tesis para optar el Título de Biólogo Acuicultor. Universidad Nacional del Santa. Nuevo Chimbote, Perú. 65p.

Jiang, X.M. (2002). Effects of temperatures, light intensity and nitrogen concentrations on the growth and fatty acid composition of Nannochloropsis oculata. Mar. Science. 26:912.

Loayza, A. (2002). Diagnóstico del humedal de Villa María. Instituto Ambientalista Natura. Chimbote, Ancash, Perú. 218p.

Ma, H.F.; X. Li; H. Hu; Y. Yu \& Y. Wu. (2012). Growth, removal of nitrogen and phosphorus, and lipid accumulation property of Scenedesmus sp. LX1 in aquaculture wastewater. Huan Jing Ke Xue. 33(6):1891-1896.

Mallick, N. (2002). Biotechnological potential of immobilized algae for wastewater N, P and metal removal: A review. Biometals. 15:377-390.

Marsh, J. \& D. Weinstein. (1966). Simple charring method for determination of lipids. J. Lipid Res. 7:574-576.

Martínez, M.; S. Sánchez; J. Jiménez; F. Yousfi \& L. Muñoz. (2000). Nitrogen and phosphorus removal from urban wastewater by the microalga Scenedesmus obliquus. Bioresource Technology. 73:263-272.

Méndez, L.; I. Albarracin; M. Cravero \& R. SaIomón. (2010). Crecimiento de Scenedesmus quadricauda en efluentes cloacales de la ciudad de Trelew, Chubut, Argentina. <http:// hdl.handle.net/1834/3639>. Accesado: 17 de abril del 2014.

Merino, F. (1999). Efecto del ácido acético con sustrato limitante en el crecimiento de Scenedesmus acutus usando cultivos batch. Informe de Investigación. Universidad Nacional del Santa. Nuevo Chimbote, Perú.

Merino, F.; W. Capa \& G. Alayo. (2003). Efecto combinado de la fuente nitrogenada y la concentración de silicato en el crecimiento y contenido de lípidos y carbohidratos de Chaetoceros gracilis en laboratorio. Informe de investigación. Universidad Nacional del Santa. Chimbote - Perú. 32p. 
Morató, J.; A. Subirana; A. Gris; A. Carneiro \& R. Pastor (2006). Tecnologías sostenibles para la potabilización y el tratamiento de aguas residuales. Revista Lasallista de Investigación. 3(1):19-29.

PNUMA. (2004). Lineamientos sobre el manejo de aguas residuales municipales. Programa de las Naciones Unidas para el Medio Ambiente (PNUMA). La Haya, Países Bajos. 112p.

Rasoul, S.; N. Montazeri; S. Shaker; A. Safari; A. Kazemi; P. Mousavi; M. Ali \& Y. Ghasemi. (2014). Removal of nitrogen and phosphorus from wastewater using microalgae free cells in batch culture system. Biocatalysis and Agricultural Biotechnology. 3:126-131.

Richmond, A. \& E.W. Becker. (1986). Technological aspects of mass cultivation - A general outline. In: Richmond A (ed.), CRC Handbook of microalgal mass culture. CRC Press Inc., Boca Raton. 245-264pp.

Shin, D.; H. Cho; J. Utomo; Y. Choi; X. Xu \& J. Park. (2015). Biodiesel production from Scenedesmus bijuga grown in anaerobically digested food wastewater effluent. Bioresour Technol. 184:215-221.

SUNASS. (2005). Informe de Supervisión a SEDACHIMBOTE S.A.: Supervisión y fiscalización desde la sede a SEDACHIMBOTE S.A. SUNASS INFORME No 156-2005/SUNASS-120-F. 50p.

Valdez, E. \& A. Vázquez. (2003). Ingeniería de los Sistemas de Tratamiento y Disposición de Aguas Residuales. Fundación ICA, A.C. México D.F., México. 341 p.

Wong, Y.; K. Yung; Y. Tsang; Y. Xia; L. Wang \& K. Ho (2015) Scenedesmus quadricauda for nutrient removal and lipid production in wastewater. Water Environ. Res. 87(12):2037-2044.

Yang, J.; M. Xu; X. Zhang; Q. Hu; M. Sommerfeld \& Y. Chen. (2011). Life-cycle analysis on biodiesel production from microalgae: Water footprint and nutrients balance. Biores. Technol. 102:159-165.

\section{CORRESPONDENCIA:}

Dr. Juan Fernando Merino Moya

fmerino@uns.edu.pe 